\title{
Synchrotron-sideband snake depolarizing resonances ${ }^{e}$
}

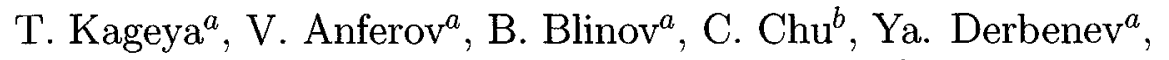 \\ A. Krisch ${ }^{a}$, S. Lee ${ }^{b}$, W. Lorenzon ${ }^{a}$, T. Rinckel ${ }^{b}$ H. Sato ${ }^{c}$, \\ P. Schwandt ${ }^{b}$, D. Sivers ${ }^{a}$, K. Sourkont ${ }^{a}$, F. Sperisen ${ }^{b}$, \\ B. von Przewoski ${ }^{b}$, V. Wong ${ }^{a}$, S. Youssof ${ }^{a}$
}

\begin{abstract}
We recently created a snake depolarizing resonance using an if solenoid magnet in a ring containing a nearly $100 \%$ Siberian snake. We found that the primary snake rf resonance also had two weaker synchrotron sidebands, which are second-order snake resonances; they were probably caused by the energy-dependent strength of the solenoid snake due to the Lorentz contraction of its longitudinal $\int B \cdot d l$. This was the first observation of an $\mathrm{rf}$ synchrotron-sideband depolarizing resonance in the presence of a nearly full Siberian snake. ${ }^{[d]}$

[a] Randall Lab of Physics, University of Michigan, Ann Arbor, MI 48109-1120, USA

[b] Indiana University Cyclotron Facility, Bloomington, IN 47408-0768, USA

[c] KEK, High Energy Accelerator Research Organization, Tsukuba, 305-0801, Japan

$[d]$ B.B. Blinov et al., Phys. Rev. ST-AB 2, 064001 (1999)

$[e]$ Supported by research grants from the U.S. Department of Energy and U.S. NSF
\end{abstract}

\section{Beam-line Polarimeter for Intermediate-Energy Deuteron}

\author{
T. Uesaka,${ }^{a}$ H. Sakai,${ }^{b}$ H. Okamura, ${ }^{a}$ A. Tamii,${ }^{b}$ Y. Satou, ${ }^{c}$ \\ N. Sakamoto, ${ }^{c}$ T. Ohnishi, ${ }^{c}$ T. Wakasa, ${ }^{d}$ K. Itoh,${ }^{e}$ K. Sekiguchi, ${ }^{b}$ \\ K. Yako, ${ }^{b}$ K. Suda ${ }^{a}$ S. Sakoda,${ }^{b}$ \\ ${ }^{a}$ Department of Physics, Saitama University, Saitama 338-8570, Japan \\ ${ }^{b}$ Department of Physics, University of Tokyo, Hongo, Bunkyo, Tokyo 113-0033, Japan \\ ${ }^{c}$ RIKEN (The Institute of Physical and Chemical Research), Saitama 351-0198, Japan \\ ${ }^{d}$ Research Center for Nuclear Physics, Osaka University, Osaka 567-0047, Japan \\ ' Tandem Center, Tsukuba University, Ibaraki 305-8577, Japan
}

We have developed a beam-line polarimeter for intermediate energy deuterons at RIKEN Acclearator Research Facility. The $d+p$ elastic scattering is used as polarimetry. Recently, calibration measurement has been carried out at $E_{d}=140$ and $200 \mathrm{MeV}$. The values of $A_{y}\left(A_{y y}\right)$ are $-0.519 \pm 0.005(0.541 \pm 0.005)$ and $-0.332 \pm 0.005(0.306 \pm 0.006)$ at $140 \mathrm{MeV}$ and $200 \mathrm{MeV}$, respectively.

CP570, SPIN 2000, $14^{\text {th }}$ International Spin Physics Symposium, edited by K. Hatanaka et al. (C) 2001 American Institute of Physics 0-7354-0008-3/01/\$18.00 